\title{
Metodología para la evaluación de impacto de la extensión en la educación superior ${ }^{1}$ \\ Methodology for the impact assessment of extension in higher education
}

\author{
DOI: http://dx.doi.org/10.17981/econcuc.39.1.2018.09 \\ Artículo de investigación. Fecha de recepción: 19/09/2017 Fecha de aceptación: 20/06/2018 \\ Carolina Rincón Zapata \\ Universidad Católica de Oriente, Rionegro (Colombia). \\ crincon@uco.edu.co \\ Adriana Pérez Cadavid \\ Universidad Católica de Oriente, Rionegro (Colombia). \\ aperez@uco.edu.co
}

Para citar este artículo:

Rincón, C. y Pérez, A. (2018). Metodología para la evaluación de impacto de la extensión en la educación superior. Económicas CUC, 39(1). 137-152. DOI: http://dx.doi.org/10.17981/econcuc.39.1.2018.09

\section{Resumen}

Las instituciones de Educación Superior (IES) desempeñan un papel fundamental en el desarrollo de estrategias orientadas a la trasformación social. El objetivo de esta investigación es proponer una metodología para evaluar las externalidades de las IES como efecto de intervenciones en sus grupos de interés desde la extensión y proyección social. La metodología fue descriptiva-documental; a partir de las categorías que determinan la transformación social, se plantearon variables que harán parte del modelo econométrico de regresión para dichas categorías, que determina un índice de impacto. El resultado son los cuatro (4) momentos de la metodología propuesta. Cada variable podrá valorarse para determinar el impacto real de un proyecto.

Palabras clave: impacto, evaluación, extensión universitaria.

\section{Abstract}

Higher Education Institutions (HEIs) play a fundamental role in the development of strategies aimed at social transformation. The objective of this research is to propose a methodology to evaluate the externalities of HEIs as an effect of interventions in their interest groups from the extension and social projection. The methodology was descriptivedocumentary; based on the categories that determine social transformation, variables were considered that will be part of the regression econometric model for these categories, which determines an impact index. The result is the four (4) moments of the proposed methodology. Each variable can be assessed to determine the real impact of a project.

Keywords: impact, evaluation, university extension.

$1 \mathrm{El}$ artículo se desprende del proyecto de investigación "Conceptos y metodologías para desarrollar evaluaciones de impacto de la internacionalización, la extensión y los egresados en la educación superior". 


\section{Introducción}

La educación universitaria según Alvarado, Hernández y Chumaceiro (2010), ha sido objeto de múltiples estudios por organismos e instituciones internacionales, tales como; la UNESCO - IESALC, el Banco Interamericano de Desarrollo y el Banco Mundial, entre otros. Esta realidad no escapa a la colombiana. La educación superior es uno de los sectores estratégicos que puede aportar al desarrollo económico y social de Colombia. El Plan Nacional de DesarrolloPNUD, 2014-2018 (República de Colombia, 2014) considera a la educación como uno de sus tres pilares fundamentales para el logro de la equidad y la paz en el país. De la misma manera, el Acuerdo 2034 de 2014 como propuesta de Política Pública para la excelencia de la educación superior en Colombia en el escenario de la paz, reconoce a la educación superior como pilar sobre el cual los colombianos podrán construir una sociedad en paz (justa, culta, democrática, solidaria, próspera, competitiva e incluyente), en la que convivan digna y pacíficamente ciudadanos libres, éticos, responsables y productivos (Consejo Nacional de Educación Superior-CESU, 2012).

Lo anterior adquiere mayor relevancia si se toma en cuenta que como lo expresa Gutiérrez (2014): "los entes territoriales y la estructura descentralizada del país deben ofrecer fundamentos para que la legislación y la política... tengan amparo desde diferentes concepciones" (p. 166). Aunado a ello, es importante comprender que hoy en día la sociedad exige acciones de transformación social, no solo a las empresas, instituciones gremiales y sector público, sino también a las universidades. Con tales acciones el país espera afrontar la crisis económica y social, financiera y de identidad, que ha traído notorias consecuencias sociales, políticas y culturales (Salinas y Osorio, 2012). El mundo actual, entonces, demanda soluciones que permitan el mejoramiento de las condiciones de vida, tanto a escala económica como social, laboral, familiar y cultural (Arcos, Suárez y Zambrano, 2015).

Ahora bien, el impacto positivo en las condiciones de vida y el bienestar de la comunidad, conocido como transformación o cambio social, puede estar determinado por la evolución de los modelos de desarrollo socio-económico que aporten al mejoramiento del bienestar y transformen gradualmente los procesos de desarrollo de los territorios, las industrias y las personas inmersas en una comunidad determinada (Arcos et al., 2015).

Por tanto, en lo que corresponde a las instituciones de educación superior (IES), estas desempeñan un papel fundamental en el desarrollo de estrategias para impactar positivamente sobre las condiciones de vida de las comunidades, es decir de trasformación social, porque son las encargadas de formar el capital social que configura a una comunidad. Al respecto, Gómez (2013) considera que las IES, mediante la práctica pedagógica, están llamadas a ejecutar estrategias de transformación, para lo cual deben partir de la lectura del contexto social y de las tendencias del desarrollo mundial. Además de las problemáticas sociales, también pueden identificar las necesidades de las comunidades y planear intervenciones que, por medio de proyectos con impacto social, permitan darles solución y generar un determinado cambio social. De esta manera las IES avanzarán en el despliegue de sus tres funciones: docencia, investigación y extensión y proyección social.

Además, Vargas citado por Alvarado et al. (2010), expresa que este es un cambio que debe cumplirse sistemáticamente, ajustado a los requerimientos, que permitan satisfacer las necesidades y expectativas de nuestros clientes o usuarios. De esta manera las IES avanzarán en el despliegue de sus tres funciones: Docencia, Investigación y Extensión y proyección social.

Por tanto, el objetivo de este artículo es proponer una metodología para la evaluación de las externalidades, entendiéndose 
estas como los cambios positivos o negativos en el bienestar de personas o grupos, que generan las IES en la sociedad como efecto de una intervención en los grupos de interés que hacen parte del alcance de las instituciones desde su función de extensión y proyección social.

\section{Aspectos Conceptuales}

En el desarrollo de las funciones sustantivas de las IES - Docencia, Investigación y extensión - se implementan diferentes estrategias enfocadas a la formación integral de los estudiantes, desde las competencias en el ser, el saber, el hacer y el convivir; tales competencias propenden por el mejoramiento de la calidad de vida de los educandos y de su perfil profesional, para el desempeño laboral. Las estrategias de formación abarcan asesorías académicas, desarrollo de prácticas profesionales, formulación de proyectos de extensión para la proyección social y de investigación para la generación de nuevos conocimientos y desarrollos tecnológicos, entre otros; estas estrategias tienen un efecto directo e indirecto sobre las condiciones de desarrollo regional, departamental y nacional, y sobre la calidad de vida de los habitantes.

En este apartado aparece la conceptualización acerca de la función de extensión, y la evaluación de impacto. Posteriormente se mencionan las metodologías de evaluación de impacto más desarrolladas, adaptables a la función de extensión, para finalmente proponer una metodología de evaluación de impacto que puede ser apropiada por las IES en el desarrollo de su función de extensión.

\section{- El concepto de la función de extensión}

La extensión configura una de las funciones sustantivas más importantes del quehacer de las IES, pero dentro de las instituciones mismas es quizá la función más ambigua en su definición, adopción y finalidad. La Ley 30 de 1992, en su artículo 120, asume como extensión a las actividades de difusión de los conocimientos, como programas de educación permanente, cursos y seminarios; al intercambio de experiencias y actividades de servicio hacia la comunidad, que propenden por el bienestar general de la comunidad y la satisfacción de las necesidades de la sociedad (Ley 30, 1992). Según lo anterior, lo que se evidencia en las IES es que han asumido la extensión de diferentes formas que se enmarcan en las anteriores definiciones y directrices dadas en el orden nacional. No obstante, las actividades, programas y proyectos que se desarrollan dentro de esta función dependen específicamente de la naturaleza de cada institución y de cómo se conciba dentro de su misión y su proyecto educativo institucional.

Según León (2013), en los orígenes de la extensión universitaria, ésta se ha ido configurando de diferentes maneras según el período histórico o las prioridades mismas de cada institución, de forma que ha sido denominada también como proyección social, proyección universitaria, responsabilidad social universitaria, integración universitaria, extensión solidaria, entre otros. Así que de manera general se define la extensión como una función misional y sustantiva de las IES, a partir de la cual se logra una interacción entre el conocimiento, y los saberes de la academia; y las necesidades de la sociedad, las organizaciones e instituciones que hacen parte de ella.

La Red de Extensión Universitaria (Asociación Colombiana de Universidades-ASCUN, 2018) concibe la extensión como parte de la estructura académica de la universidad, a partir de la cual se busca aportar a la solución de problemas dentro de las comunidades, mediante la interacción e integración con las mismas. Adicionalmente, la extensión educativa deberá contribuir a la transformación de la sociedad, con perspectiva de democratización y equidad social, regional, política y cultural. 
Las IES materializan esta labor por medio de los contratos con empresas oficiales o privadas en trabajos de consultorías, asesorías, interventorías; diplomados, cursos libres, talleres, seminarios, conferencias presenciales de transferencia social de conocimiento; uso y divulgación del conocimiento por medios masivos de información como radio, televisión, prensa, revistas, folletos; consultorios jurídicos, psicológicos, disponibilidad de laboratorios o talleres; prácticas universitarias, pasantías universitarias, gestión cultural, entre otros (República de Colombia, Ministerio de Educación Nacional, 2013).

En la actualidad, las principales modalidades de extensión manifestadas en las IES Colombianas, según ASCUN, (2008), son: Educación para el trabajo y el desarrollo humano, servicios académicos de extensión, servicios docente-asistenciales, gestión social, gestión tecnológica, programas interdisciplinarios de extensión que integran formación e investigación, prácticas universitarias en extensión, gestión de procesos culturales, proyectos y programas de comunicación pública y de difusión educativa y cultural, gestión de relaciones con los egresados y las intervenciones de docentes en eventos externos.

A partir de lo anterior, el desarrollo de las actividades bajo las cuales se manifiesta la función extensión en las diferentes IES, se asemeja a un ejercicio que metodológicamente implica el desarrollo por proyectos, adoptando dentro de todos los procesos y actividades la planeación, ejecución, verificación y actuación. Esto último requiere no solamente el planteamiento de unos objetivos claros, sino también la medición de indicadores que puedan evidenciar el cumplimiento de los objetivos trazados, y el impacto de éstos en la sociedad a lo largo del tiempo.

Entendida, entonces, la función de extensión como el desarrollo de proyectos de tipo social, se encuentran múltiples acepciones de la evaluación de impacto, no muy diversas.
Por ejemplo, Salamanca (1995) define la evaluación de impacto como aquella que se fija en indagar en los efectos secundarios o colaterales de una intervención social cualquiera, englobando sus externalidades positivas y negativas, efectos directos y secundarios de tipo diferido en el tiempo y que se realiza en dos momentos específicos del ciclo de vida de la intervención social.

Por su parte, Valdés (2008), quien parte de la premisa de que toda intervención social busca modificar exitosamente la situación problema que motivó dicha intervención, considera a la más actual evaluación de impacto de proyectos sociales como aquella que intenta constatar los cambios producidos por las intervenciones a que son sometidas las poblaciones objetivo en la cual es aplicado un cierto proyecto.

Los proyectos que generan cambios o transformaciones sociales, pueden estar destinados a satisfacer directamente necesidades sociales de una comunidad mediante un servicio, generar planes de transformación productiva, ser instrumento para desencadenar actividades productivas que mejoren los ingresos y las condiciones de vida de la comunidad, o producir y fortalecer las capacidades de la comunidad con el fin de generar beneficios directos e indirectos en el mejoramiento de la calidad de vida (Miranda, 1997).

\section{- El concepto de evaluación de impacto}

La evaluación hace referencia a un juicio de valor, un señalamiento, un estimativo, una apreciación, una medida de algo. La evaluación va adquiriendo su significado según al ámbito en que se aplique: académico, investigativo, social, ambiental, político, tecnológico, institucional, entre otros.

Tyler (citado por Mora, 2004) considera que la evaluación es el proceso que determina en qué medida se han logrado los objetivos, para lo cual recomienda la comparación de los resultados y los objetivos inicialmente planteados; esto a su vez proporciona una 
realimentación para identificar cuáles han sido los aspectos más relevantes que se están dando en el proceso, para alcanzar con éxito lo propuesto.

Por su parte, el término impacto, de acuerdo con el Diccionario de uso del español proviene de la voz impactus, del latín tardío y que significa, en su tercera acepción, impresión o efecto muy intensos dejados en alguien o en algo por cualquier acción o suceso.

En este sentido, el término impacto está asociado a los cambios, las transformaciones o los efectos positivos o negativos que se generan en el bienestar de las personas o los grupos, como resultado de la intervención a una necesidad que se identifica y se le pretende cambiar de condición. A partir de esta definición se puede decir que el impacto de una acción se reconoce siempre y cuando genere un cambio social.

A su vez, el impacto se identifica, generalmente, con los cambios en el ser, hacer, saber o tener de las personas, familias, grupos, comunidades o regiones; cambios que a su vez afectan, explican o determinan unas condiciones sociales, económicas, políticas, culturales, y/o ambientales (Quintero, 1995).

En cuanto al concepto de evaluación de impacto, la Asociación Internacional de Evaluación de Impactos (IAIA, 2012) afirma que es un proceso que tiene como objetivo identificar las consecuencias futuras de una acción actual o propuesta. El impacto, así entendido, es la diferencia entre lo que pasaría con la acción y lo que ocurriría sin ella.

En lo que concierne a la educación, el impacto se asocia a la identificación de los alcances logrados, en términos del efecto, la transformación, los cambios esperados y logrados derivados de una intervención (Aponte, 2007). Ahora bien, en la educación superior se pueden identificar múltiples intervenciones que efectúa la institución en el ejercicio de las funciones de investigación, docencia y proyección social, las cuales generan cambios y transformaciones; pero en el presente apartado se focalizan solo las intervenciones realizadas desde el ejercicio de la extensión, específicamente mediante el desarrollo de proyectos que parten de la necesidad o problema identificado en alguno de los grupos de interés de las IES, los cuales pueden tener un alcance en los ámbitos productivo, cultural, social, ambiental y político.

\section{- Fases para la gestión de proyectos con impacto social}

Un proyecto de tipo social parte de la identificación de un problema o necesidad y se fundamenta en los aspectos principales que lo caracterizan, como aspectos sociales, económicos, políticos, localización de la población afectada, antecedentes de evolución del problema, consecuencias que está generando el problema y aquellas que podrá seguir generando en caso de no dársele solución. A partir de esto, se pueden plantear posibles alternativas de solución, que finalmente se materializan en la formulación de un proyecto (Miranda, 1997).

La segunda etapa del ciclo del proyecto se refiere a la ejecución del mismo, que consiste en llevar a cabo las actividades planeadas con las que se espera dar respuesta a la problemática, disponiendo los recursos humanos, técnicos, administrativos y financieros necesarios para tal fin. En esta etapa se audita continuamente la ejecución de las actividades y la evolución de la población beneficiada.

Finalmente, la tercera etapa del proyecto se refiere al proceso de evaluación del mismo. Como se menciona en párrafos anteriores, existen varios tipos de evaluación, pero el interés del presente apartado es referir la metodología de evaluación de impacto, con el fin no solo de plantear un nivel de evaluación microeconómico, que se remita al estudio de indicadores propios del proyecto, sino también de auscultar los efectos macroeconómicos del proyecto en el 


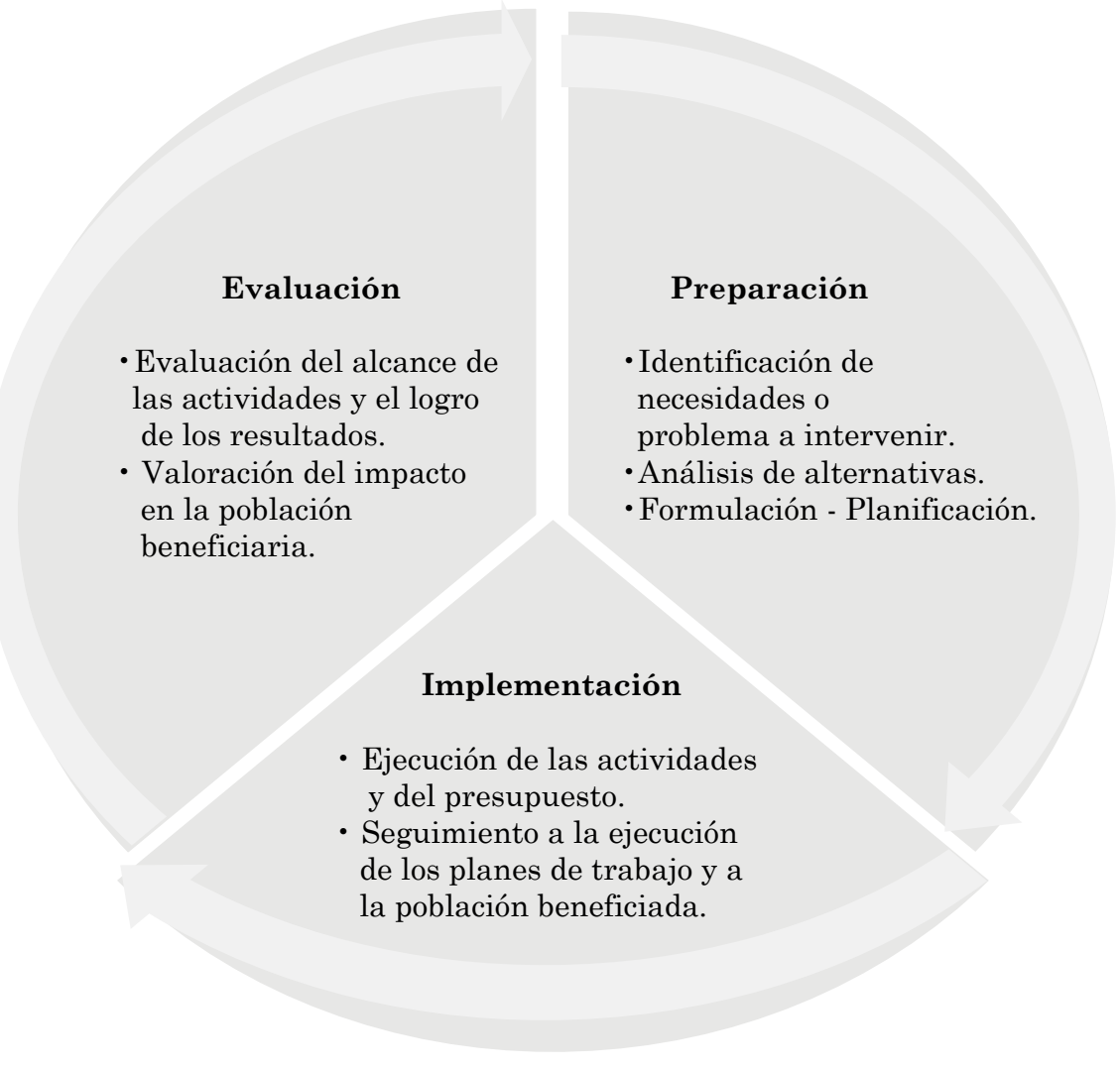

Figura 1. Fases para la gestión de proyectos con impacto social.

Fuente: elaboración propia a partir de González (2000).

entorno o grupos de interés. La figura 1 representa las tres etapas descritas anteriormente del ciclo del proyecto.

\section{- Metodologías para la evaluación de impacto}

Existen diferentes metodologías para evaluar el impacto de los programas desarrollados por las organizaciones. La mayoría de metodologías responden a proyectos de tipo social, donde lo que se pretende es determinar si los efectos ocasionados con el desarrollo de los programas son los efectos deseados en los grupos poblacionales, si son realmente efectos ocasionados a partir de la intervención programada, y si además existen externalidades positivas o negativas en los grupos de interés, entendiendo por externalidad las consecuencias no atribuidas al objetivo del proyecto.

Para lograr lo anterior se debe, como parte de la evaluación de impacto, identificar y separar los resultados logrados como consecuencia de la intervención, de aquellos resultados que de todas maneras habrían ocurrido sin la ejecución del programa y que sean atribuidos a la evolución normal de los individuos o de las condiciones del entorno. Lo anterior implica no solo construir o identificar las variables que serán evaluadas para determinar el impacto del programa, sino que, además, deberá considerarse cómo evaluar el efecto sobre los individuos con o sin la intervención del programa (Berner, Cooper, Guzmán y Guzmán, 2004). 
Posteriormente, para evaluar el efecto de un programa sobre los individuos se hace necesario determinar en los resultados el efecto causal del programa sobre los mismos. Gertler, Martínez, Premand, Rawlings y Vermeersch (2011) se refieren al efecto causal cuando se logra establecer en qué medida un programa específico, y solo ese programa, ha contribuido a cambiar un resultado; los autores descartan la posibilidad de que cualquier factor diferente del programa explique el impacto observado. De tal forma que el impacto causal de un programa sobre un resultado sería la diferencia entre el resultado con el programa y el mismo resultado sin el programa.

Esto conduce a la necesidad de estimar qué ocurriría con las personas o grupos de interés si no hubiesen sido partícipes o beneficiarios del programa, esto para establecer la diferencia entre los resultados con y sin programa. Baker (2000) resalta la necesidad que toda evaluación de impacto posee de estimar el escenario contrafactual o simulado alternativo, que no es más que lo que habría ocurrido si el proyecto nunca se hubiera realizado o lo que habría ocurrido normalmente.

La estimación del escenario contrafactual se logra mediante el establecimiento de grupos de control o de comparación, que es posteriormente comparado con el grupo de tratamiento, el cual incluye a las personas que efectivamente reciben o se benefician de la intervención.

Por tanto, el objetivo de la evaluación de impacto se convierte en encontrar el mejor grupo de control, que realmente posea semejanzas con el grupo de tratamiento, y que la única diferencia que puedan reflejar sea la participación en los programas. Gertler et al. (2011) definen la identificación de esos grupos de comparación como la esencia de cualquier evaluación de impacto, independientemente del programa que se evalúe, lo que conduce a que sin una estimación válida del contrafactual no se pueda conocer el verdadero impacto de un programa.

Considerando como principal objetivo la determinación de los grupos de tratamiento y de control, seguido del método de análisis de los resultados, las principales metodologías desarrolladas para la evaluación de impacto son: cuantitativas, cualitativas y mixtas. A continuación se describen estas metodologías, basadas en una revisión documental de diferentes autores como Baker (2000), Mohr (1995), Valadez y Bamberger (1994), Gertler et al. (2011) e Imbens y Wooldridge (2009); el criterio de revisión fue que los contenidos sean aplicables a la presente investigación.

Las metodologias cuantitativas buscan determinar el grupo de participantes del programa (grupo de tratamiento), y el grupo de no participantes (grupo de control), estadísticamente idénticos en ausencia del programa. El uso de las metodologías dependerá del objeto de cada programa, de la pregunta que mediante la evaluación de impacto se quiera resolver y de las reglas para la inscripción de los participantes de un programa.

Según Baker (2000), las metodologías cuantitativas tradicionalmente utilizadas se basan en diseños experimentales y diseños cuasi experimentales. Los primeros, además de tener claridad acerca del objeto del proyecto y el grupo de beneficiarios, exigen de forma anticipada distribuir de manera aleatoria la intervención dentro del grupo de beneficiarios. En consecuencia, durante la ejecución del proyecto se cuenta con un grupo beneficiario real del proyecto (grupo de tratamiento), a los que se puede constantemente realizar seguimiento mediante la medición de las variables a estudiar, para ser finalmente contrastado con las variables arrojadas por beneficiarios potenciales no intervenidos, quienes constituyen el grupo de control. 
En cuanto a las metodologías cualitativas, Mohr (1995) señala que estas metodologías para evaluación de impacto son precisas para determinar el efecto de las intervenciones, basándose en algo diferente al contrafactual, pero sin dejar de establecer la relación causal que tienen los proyectos sobre los resultados en los grupos de interés. Estas metodologías buscan comprender, de una manera guiada, cómo los individuos o grupos estudiados cambian sus comportamientos, formas de comprender y las condiciones, a partir de la intervención realizada. Para estos casos no existe grupo de control.

Shahidur, Gayatri y Hussain (2010) consideran que las metodologías cualitativas pueden convertirse en herramienta para entender los mecanismos a través de los cuales el programa ayuda a los beneficiarios, porque estas se basan en la recolección de información descriptiva entre quienes configuran el grupo de tratamiento. Se considera información descriptiva aquella que incorpora la visión de los beneficiarios en la y de la evaluación, y que ayuda a entender y evaluar los procesos sociales que se tejen alrededor del grupo poblacional intervenido, como conflictos suscitados en torno al programa, razones por las cuales los beneficiarios no usan los servicios ofrecidos, comportamientos organizacionales como cultura, clima organizacional, entre otros (Berner et al., 2004).

Por último, las metodologías mixtas plantean una integración de la cuantitativa y la cualitativa. En un intento de comparar ambos métodos, varios autores concluyen que el uno no excluye al otro, y que además su uso deberá ser integrado. Según Baker (2000), las evaluaciones de impacto realizadas a partir de metodologías cuantitativas son más adecuadas para evaluar la causalidad, porque consideran la diferencia de los resultados obtenidos entre los grupos de tratamiento y de control; mientras que los métodos cualitativos brindan información más profunda sobre las perspectivas de los beneficiarios y los motivos de algunos resultados.

Por tanto, la integración de las dos metodologías proporcionará resultados más amplios, rigurosos y profundos toda vez que, por ejemplo, los métodos cualitativos se pueden usar para informar las variables o cuestionamientos claves para la evaluación del impacto, y los métodos cuantitativos se pueden usar para informar las estrategias de recopilación de datos, para el diseño muestral y para controlar las características no observables de los beneficiarios de los proyectos (Shahidur et al., 2010).

Una verdadera evaluación de impacto se distingue precisamente por la aplicación de las metodologías expuestas. Dada su complejidad y retos, una institución de educación superior debe sopesar la pertinencia y conveniencia de aplicar una evaluación de impacto en la función de extensión, descartando primero otro tipo de evaluación.

\section{Aspectos MetodologÍcos}

La metodología bajo la cual se desarrolló la investigación es, en su parte inicial, descriptiva-documental puesto que abarcó la descripción, análisis e interpretación de la naturaleza actual de las evaluaciones de impacto existentes y la comprensión de este tipo de procesos. Para ello se realizó un rastreo documental tanto de las evaluaciones de impacto existentes en las IES, en cuanto a su función de extensión, como de las evaluaciones de impacto no específicas de educación superior y de las categorías que podrían ser consideradas para la identificación del cambio social o transformación social.

Posteriormente, a partir de las categorías que determinan la transformación social, se plantearon las variables que podrán hacer parte de un modelo econométrico de regresión que corresponda a dichas categorías y que determina un índice de impacto. 
Diseño de la evaluación

Determinación y construcción de las variables e indicadores para medir el impacto: Podrán existir variables cualitativas y variables cuanitativas

\begin{tabular}{|lllll}
\multicolumn{5}{c}{ Definición de los instrumentos para recolectar la información } \\
Entrevistas $\begin{array}{l}\text { Focus } \\
\text { group }\end{array}$ Encuesta $\begin{array}{c}\text { Panel de } \\
\text { expertos }\end{array}$ & Observación & Test \\
psicológicos & Otros
\end{tabular}

Estimar el grupo de tratamiento y el grupo de control

Metodologías cuantitativas Metodologías cualitativas Metodologías mixtas

Recopilación y análisis de la información:

Pre y Post: Antes y Después de la intervención.

Deberá definirse si se dispone de la información tanto para el grupo de tratamiento, como para el grupo de control, o solo será aplicado el instrumento para el grupo de tratamiento antes y desués de la intervención o proyecto

Evaluar la diferencia del índice de impacto entre el grupo de tratamiento y el grupo de control o Evaluar la diferencia del índice de impacto antes del proyecto y después del mismo para el grupo de tratamiento

Figura 2. Fases para la gestión de la evaluación de proyectos con impacto social.

Fuente: elaboración propia.

Un modelo econométrico es un recurso que permite hacer uso de herramientas estadísticas, expresando las teorías ya sean económicas o socioeconómicas, para describir e interpretar los hechos de la realidad, y poder medir el impacto de una o más variables sobre otra (Wooldridge, 2008). Por tanto, se propone un modelo econométrico porque por medio de este es posible determinar o explicar una variable, a partir de las categorías que sean definidas.

De esta forma, por ejemplo, podrá definirse que, si se denomina como $\mathrm{X}$ al índice de impacto sobre las acciones de extensión emprendidas por las IES, este estaría determinado por indicadores que se establezcan en cada caso, Y y Z. Lo anterior se expresa mediante un modelo de regresión lineal múltiple que, según Wooldridge (2008), permite que varios factores observados afecten o determinen una variable $\mathrm{X}$, que además considera variables no posibles de explicar y errores dentro de la modelación.

\section{Resultados y Discusión}

En este apartado se presenta la propuesta metodológica para la evaluación de impacto en el ejercicio de la función de extensión de las IES. Como resultado de los anteriores conceptos y metodologías estudiadas, la metodología propuesta considera los pasos que se describen en la figura 2. 
El primer momento de la evaluación (determinación y construcción de las variables e indicadores para medir el impacto) es uno de los más importantes porque determina la calidad, pertinencia y utilidad de la evaluación de impacto. Consiste en identificar, a partir de los factores de impacto que determinan la transformación social, las variables a estudiar para, a partir de estas, construir los indicadores de impacto que respondan a los objetivos del proyecto. Las variables e indicadores relacionados con la eficacia, eficiencia, pertinencia y sostenibilidad de la intervención serán consideradas como complemento de la evaluación de impacto y no son objeto de la presente metodología.
A continuación, se proponen cinco factores de impacto, representados en un modelo de factores para la determinación de la transformación social, a partir de los cuales podrán identificarse las variables de análisis para la construcción de los indicadores.

Los factores de impacto, están determinados por aspectos relevantes propios de los proyectos, pero que evidencian el impacto de los mismos. En este sentido, para cada uno de los factores se han definido, a manera de guía, algunos aspectos (tabla 1) que pueden ser considerados de impacto.

\section{TABLA 1}

Descripción de los factores de impacto

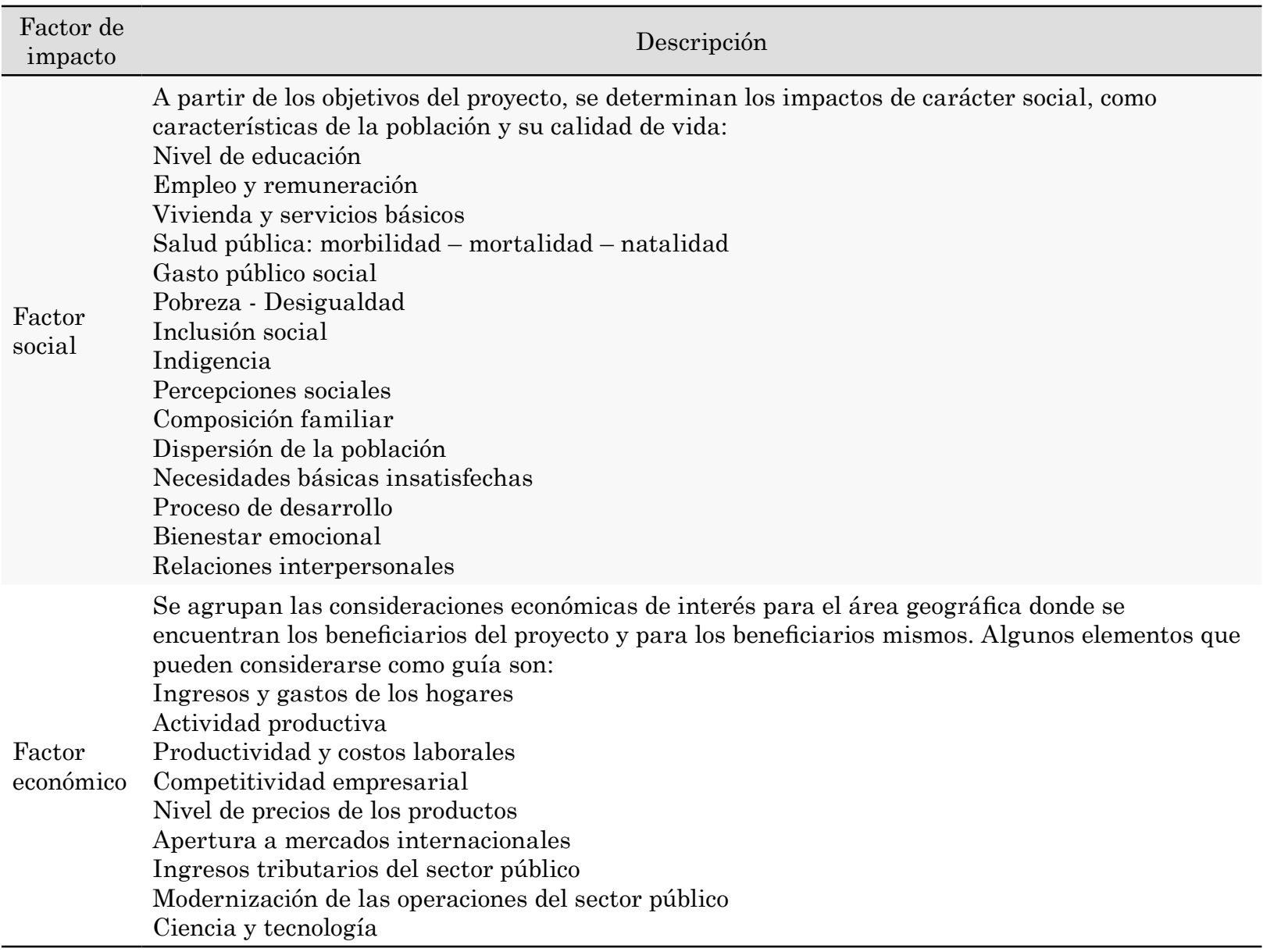




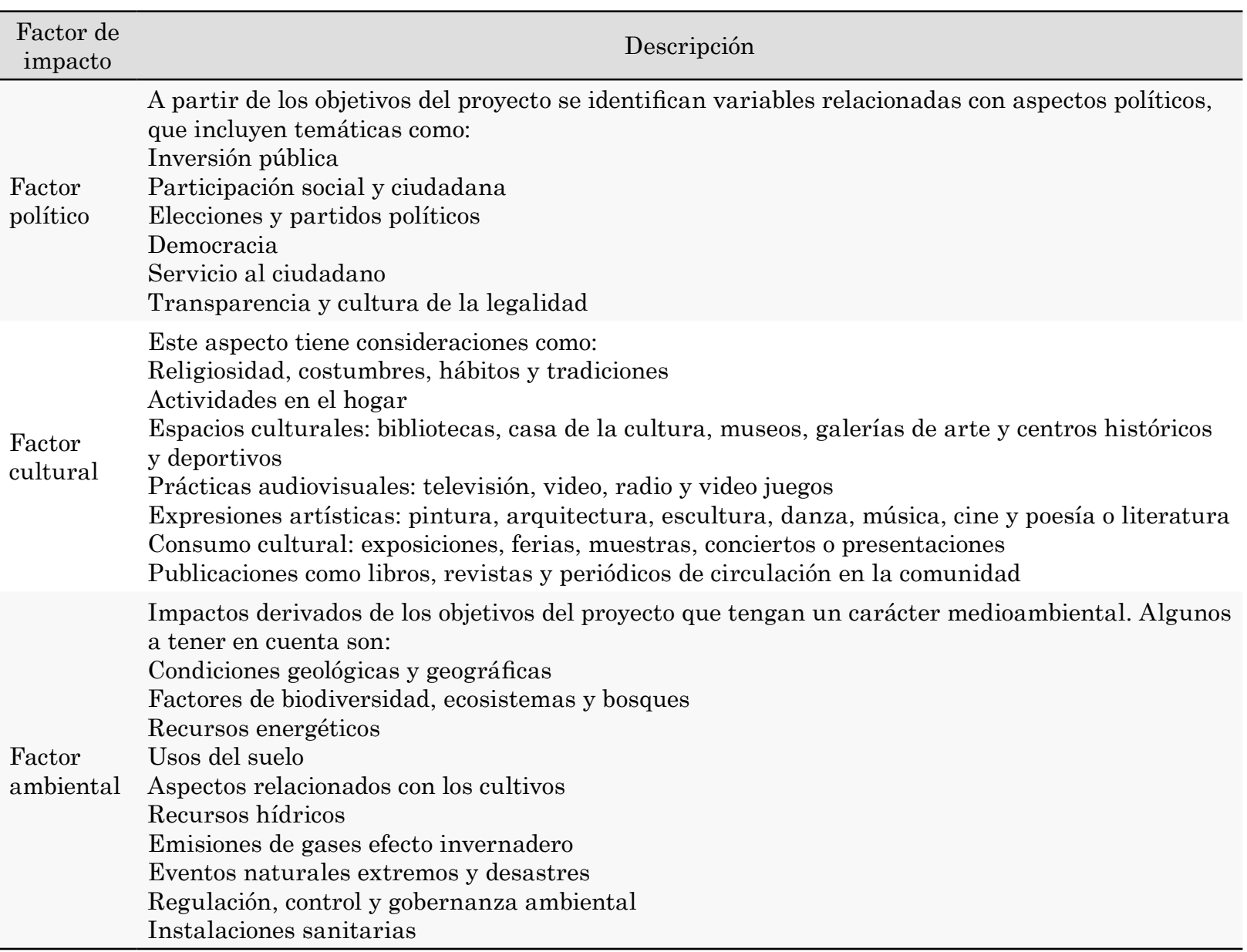

Fuente: elaboración propia a partir del Departamento Administrativo Nacional de Estadística-DANE (2015), DANE (2016) y Comisión Económica para América Latina y el Caribe-CEPAL (2017).

Es importante resaltar, en primera instancia, que las variables traducidas en indicadores, objeto de estudio o evaluación, deben ser tanto variables de tipo cualitativo como de tipo cuantitativo porque, si se quiere ser lo suficientemente riguroso, la evaluación de un programa debe abarcar ambas dimensiones de los diferentes factores objeto de estudio. Berner et al. (2004) plantea que las dimensiones cuantitativas de la evaluación se expresan mediante variables cuantitativas; sin embargo, los aspectos cualitativos también son susceptibles de mediciones cuantitativas.

Los anteriores aspectos deberán ahora definirse, representarse o agruparse, a su vez, en diferentes indicadores que deter- minen las variables de análisis. En la tabla 2 se presenta una guía de lo que debe considerar la definición de los indicadores, por medio de cuatro ejemplos a partir del factor económico, en una intervención a empresas que tiene como objetivo acompañar desde la institución a la empresa para la mejora de su productividad.

En los ejemplos que muestra la tabla 2 se pueden identificar dos variables: Gestión comercial e Indicadores de producción, que determinan para éste ejemplo el factor económico. En la primera de estas se ejemplifican tres indicadores: Plan de mercadeo, Participación en el mercado y Venta de nuevos productos; donde el primero de ellos es de tipo cualitativo y los otros son de tipo cuantitativo. 
TABLA 2

Guía para la construcción de indicadores

\begin{tabular}{|c|c|c|c|}
\hline \multicolumn{4}{|c|}{ Gestión Comercial } \\
\hline Indicador & Definición del indicador & Forma del cálculo & $\begin{array}{c}\text { Unidad } \\
\text { de medida }\end{array}$ \\
\hline $\begin{array}{l}\text { Plan de } \\
\text { mercadeo }\end{array}$ & $\begin{array}{l}\text { Indica si la empresa tiene definido un } \\
\text { planeamiento estratégico, comercial y de } \\
\text { mercadeo }\end{array}$ & $\begin{array}{l}\text { Evidencia del plan } \\
\text { comercial y de la } \\
\text { gestión del mismo }\end{array}$ & Cualitativo \\
\hline $\begin{array}{l}\text { Participación } \\
\text { en el mercado }\end{array}$ & $\begin{array}{l}\text { Es un índice de concentración de mercado } \\
\text { que tiene la empresa, respecto al total } \\
\text { de la industria, e indica la capacidad de } \\
\text { mercado de la misma }\end{array}$ & $\begin{array}{l}\text { Ingresos operacionales } \\
\text { de la empresa / } \\
\text { Ingresos operacionales } \\
\text { total de la industria }\end{array}$ & Porcentaje \\
\hline $\begin{array}{l}\text { Venta de } \\
\text { nuevos } \\
\text { productos }\end{array}$ & $\begin{array}{l}\text { Indica si la empresa es efectiva en } \\
\text { posicionar nuevos productos en el } \\
\text { mercado, y que estos sean comercializados } \\
\text { y demandados. Representa la proporción } \\
\text { de ventas de la empresa, que corresponde } \\
\text { a la demanda de nuevos productos }\end{array}$ & $\begin{array}{l}\text { Valor de venta de } \\
\text { nuevos productos / } \\
\text { Ventas totales }\end{array}$ & Porcentaje \\
\hline \multicolumn{4}{|c|}{ Indicadores de Producción } \\
\hline Indicador & Definición del indicador & Forma del cálculo & $\begin{array}{c}\text { Unidad } \\
\text { de medida }\end{array}$ \\
\hline $\begin{array}{l}\text { Crecimiento } \\
\text { en la } \\
\text { producción }\end{array}$ & $\begin{array}{l}\text { Indica que tanto han crecido o disminuido } \\
\text { las cantidades de la producción en el } \\
\text { último periodo }\end{array}$ & $\begin{array}{l}\text { 1- (Cantidad producida } \\
\text { en el periodo actual / } \\
\text { Cantidad producida en } \\
\text { el periodo anterior) }\end{array}$ & Porcentaje \\
\hline
\end{tabular}

Fuente: elaboración propia.

Para lo anterior, cada uno de los indicadores propuestos en la evaluación de impacto de cada proyecto, deberá ser estandarizado en una misma, y es éste el punto más importante en la definición de los indicadores para la construcción del índice de impacto. Para cada uno de los indicadores, deberán establecerse unos criterios de cumplimiento basados en una matriz de valoración, para obtener una medición del estado de cada uno de ellos según los datos logrados mediante la aplicación de instrumentos o información secundaria.

Es importante señalar que, desde la planificación del proyecto y de la evaluación, deberá tenerse conocimiento del ideal para cada una de las variables analizadas, de manera que la escala estable- cida, defina los rangos de cumplimiento del indicador.

Estos criterios podrán ser definidos por el grupo evaluador para cada indicador, a partir de información obtenida en fuentes secundarias y primarias, basadas estas últimas en criterios de expertos en los temas que comprenden los indicadores o sitios de información oficial.

Posterior a la matriz de valoración, deberá unificarse la escala de medición, para ubicar tanto al grupo de tratamiento como al grupo de control en un rango de medición de los factores de impacto. Además de proporcionar una idea global de la situación en la que se encuentran cada uno de los grupos, lo anterior permite comparaciones finales para determinar el impacto del proyecto. 
Para la valoración de los factores de impacto (social, económico, político, cultural y ambiental), entonces, inicialmente se realiza una valoración para cada una de las variables que los componen, donde se consideran los valores obtenidos por cada indicador, que además deberán ser ponderados con unos factores de ponderación asignados, a partir del nivel de relevancia de cada uno de los indicadores. A continuación, se realiza un ejemplo de la valoración de la variable Gestión comercial, que hace parte del factor económico en el ejemplo empleado:

Gestión Comercial $=\sum_{i=1}^{3}$ Indicador de Gestión comercial $i * \beta$

Donde:

- Indicador de Gestión comercial $1=$ Valoración determinada para el indicador denominado Plan de mercadeo

- Indicador de Gestión comercial $2=$ Valoración determinada para el indicador denominado Participación en el mercado

- Indicador de Gestión comercial $3=$ Valoración determinada para el indicador denominado Venta de nuevos productos

- $\beta$ es un factor de ponderación que deberá ser determinado según el peso que se defina para cada indicador dentro de la variable. Se recomienda hacer uso de un instrumento para ponderación de indicadores, y aplicarlo a grupos de académicos, de empresarios, comunidades y expertos.

Bajo la misma metodología deberán ser valoradas las demás variables que componen cada factor: Factor económico, social, ambiental, político y cultural. En el ejemplo referido, el factor económico está compuesto solo por dos variables: Gestión comercial e Indicadores de producción, de forma que el factor económico deberá ser valorado así:

Factor Económico $=\sum_{N=1}^{2}$ Variable $N^{*} \beta$
Donde:

- Variable $N$ se refiere a la Gestión comercial y a los Indicadores de producción

- $\beta$ es un factor de ponderación asignado para cada aspecto

Finalmente, el índice de impacto es conformado por los factores de impacto considerados, teniendo en cuenta también la ponderación que a ellos se les asigne en consecuencia directa con el tipo y objetivos del proyecto o programa.

Lo anterior, se podrá ver reflejado en un modelo de Regresión Múltiple, ya que por medio de éste es posible determinar o explicar una variable, a partir de las categorías que sean definidas. De ésta forma, por ejemplo, podremos definir que, si denominamos como variable dependiente "Y", al índice de impacto sobre las acciones de extensión emprendidas por las IES, éste estaría determinado por los factores ya definidos, así: $\mathrm{X}_{1}$ : Factor Social; $\mathrm{X}_{2}$ : Factor Económico; $\mathrm{X}_{3}$ : Factor Político; $\mathrm{X}_{4}$ : Factor Cultural; $\mathrm{X}_{5}$ : Factor Ambiental.

De lo anterior, el modelo de regresión propuesto, podrá ser expresado así:

Índice de Impacto $=\beta_{1}$ * Indicador de Factor Social $+\beta_{2} *$ Indicador de Factor Económico $+\beta_{3} *$ Indicador de Factor Político $+\beta_{4} *$ Indicador de Factor Cultural $+\beta_{5} *$ Indicador de Factor Ambiental+Error

Donde

- $\beta_{1}, \beta_{2}, \beta_{3}, \beta_{4}, \beta_{5}$, son las ponderaciones de cada indicador en el global, es decir en el índice de impacto. Se proponen con una proporción equitativa, es decir un peso de 0.2 a cada factor, y posteriormente deberán iterase hasta encontrar un modelo que mejor se ajuste al índice de impacto, a partir de las observaciones realizadas. El ajuste del modelo, se evalúa a partir del coeficiente de determinación $\mathrm{R}^{2}$

- Error experimental y aleatorio: Se refiere a las fluctuaciones de los factores individuales que no pueden ser explicadas. 
Finalmente, lo anterior cobra validez siempre y cuando sea aplicada al menos en dos momentos (un momento para el grupo de tratamiento y otro para el grupo de control) en caso de solo realizar una evaluación ex post, es decir al finalizar el programa, con el fin de lograr la evaluación del impacto del mismo. De manera que el impacto del programa representado con $a$, es:

$$
\begin{aligned}
\alpha= & \text { Índice de impacto para el } \\
& \text { grupo de tratamiento - } \\
& \text { Índice de impacto para } \\
& \text { el grupo de control }
\end{aligned}
$$

\section{Conclusiones}

El cambio social ocupa ahora una de las principales líneas de acción de los planes estratégicos municipales, nacionales e internacionales. El cambio social, entendido también como la transformación social, puede ser inducido por medio del desarrollo de programas y proyectos con impacto social.

Los principales factores que determinan el impacto social o la transformación social, inducida por la ejecución de proyectos, se relacionan con el mejoramiento de la calidad de vida de las personas y de las condiciones del entorno en que estas se desenvuelven. Estos factores están definidos como: factores sociales, económicos, políticos, culturales y ambientales. No obstante, el ejecutor de los proyectos podrá considerar otros factores y ser incluidos en la metodología para la definición de variables y construcción de indicadores que determinan el impacto del proyecto.

Las IES se convierten en agentes determinantes para la transformación social y esta deberá ser concebida como parte de la naturaleza del ejercicio de su función de extensión, por medio de la cual las IES no solo llevan a la sociedad el conocimiento y los desarrollos investigativos sino que también ejecutan proyectos acordes a las necesidades y problemáticas sociales.

La evaluación del impacto que el desarrollo de estos proyectos tiene en el cambio social puede realizarse por medio de la metodología de evaluación de impacto propuesta en este artículo, donde a partir de los objetivos propios de cada programa se determinan unos indicadores de impacto que hacen posible la medición de variables cualitativas y cuantitativas y que además definen un índice de impacto cuantificable a partir de los factores del cambio social. Finalmente, la diferencia del índice de impacto entre la población beneficiada y el contrafactual evaluará el impacto del proyecto.

El objetivo de una evaluación de impacto es obtener conocimiento acerca del grado de incidencia de un proyecto en las personas o el contexto al cual ha sido dirigido, esto para mejorar futuros desarrollos, réplicas o rediseñar proyectos y programas. Adicionalmente, sirven para establecer los aspectos positivos y negativos de la intervención, demostrar los beneficios de las relaciones entre la institución de educación superior y la sociedad y contribuir a configurar políticas públicas. En tanto los retos de una evaluación de impacto son tan profundos y numerosos los propósitos y funciones, estos deben ser tan significativos que se justifique su aplicación.

\section{Referencias}

Alvarado, L., Hernández, J. y Chumaceiro, A. (2010). Calidad de servicio en universidades públicas venezolanas. Revista TEAC'S, 4(2), 45-58. Recuperado de https://dialnet.unirioja.es/servlet/ articulo?codigo $=3991416$

Aponte, C. (2007). Conceptos básicos para el análisis y la evaluación de impacto en la Universidad de la Sabana. Chía: Universidad de la Sabana. 
Arcos, C., Suárez, M., y Zambrano, S. (2015). Procesos de innovación social (IS) como fuente de transformación social de comunidades rurales. Revista Academia y Virtualidad, 8 (2), 8599. https://doi.org/10.18359/ravi.1425

ASCUN. (2018). Políticas de Extensión. Recuperado de https://www.ascun.org. co/uploads/default/networks/73a4479a 5d33fb6eb23fc83e7c0ee514.pdf

ASCUN. (19 de febrero de 2008). Por medio del cual se adopta El II Documento de trabajo para políticas de extensión. [Acuerdo 2]. Recuperado de https://www.ascun.org.co/uploads/default/networks/acc39556d2b5746e3dba8fdd41d12c7a.pdf

Baker, J. (2000). Evaluación del impacto de los proyectos de desarrollo en la pobreza. Manual para profesionales. Washington, D.C.: Banco Mundial. Recuperado de http://siteresources. worldbank.org/INTISPMA/Resources/Impact-Evaluation-Handbook-Spanish-/manual.pdf

Berner, H., Cooper, R., Guzmán, M. y Guzmán, N. (2004). Metodología Evaluación de Impacto. Santiago: Gobierno de Chile.

CEPAL (2017). Panorama regional de América Latina y el Caribe: Indicadores seleccionados. Recuperado de http:/estadisticas.cepal.org/cepalstat/ WEB_CEPALSTAT/Portada.asp

CESU (2012). Acuerdo por lo superior 2034. Recuperado de http://www.mineducacion.gov.co/1621/articles-344500_Brochure_acuerdo_Superior.pdf

DANE (2016). Boletín técnico: Encuesta de Consumo Cultural -ECC. Recuperado de https://www.dane.gov.co/files/ investigaciones/eccultulral/bole_ ecc_2016.pdf
DANE (2015). Boletín técnico: Encuesta de cultura política. Recuperado de https://www.dane.gov.co/files/investigaciones/ecpolitica/bol_ECP_15.pdf

Gertler, P., Martínez, S., Premand, P., Rawlings, L. y Vermeersch, C. (2011). La evaluación de impacto en la práctica. Washington, D.C.: Banco Mundial. Recuperado de http://siteresources. worldbank.org/INTHDOFFICE/Resources/ I E P_S PA N I S H _ F I . NAL_110628.pdf

Gómez, D. (2013). A propósito de la reflexión de las prácticas pedagógicas y la calidad de educación. Uni-pluri/versidad, 13(2), 119-122. Recuperado de http://aprendeenlinea.udea.edu.co/revistas/index.php/unip/article/download/16993/14713

González, L. (2000). La evaluación ex-post o de impacto: Un reto para la gestión de proyectos de cooperación internacional al desarrollo. Cuadernos Hegoa, (29). 19-36. Recuperado de http://www. ehu.eus/ojs/index.php/hegoa/article/ download/10792/10066

Gutiérrez, J. (2014). Costos sociales de transacción de la Ley de Restitución de Tierras en Colombia: Un país sin Reforma Agraria. Jurídicas CUC, 10 (1), $157-196$.

IAIA. (2012). Evaluación de Impactos. Recuperado de http://www.iaia.org/pdf/ Fastips_1-Impact-Assessment-Sp.pdf

Imbens, G. y Wooldridge, J. (2009). Recent Developments in the Econometrics of Program Evaluation. Journal of Economic Literature, 47(1), 5-86.

León, A. (2013). Caracterización de la extensión solidaria en la Universidad Nacional de Colombia. [Tesis magistral]. Universidad Nacional de Colombia, Bogotá, D.C. Recuperado de http://bdigital. unal.edu.co/45404/1/80759477.2013.pdf 
Miranda, J. (1997). Gestión de Proyectos: Identificación, Formulación y Evaluación. Bogotá, D.C.: MB.

Mohr, L. (1995). Impact Analysis for Program Evaluation. Newbury Park: Sage.

Mora, A. (2004). La evaluación educativa: Concepto, períodos y modelos. Revista Electrónica Actualidades Investigativas en Educación, 4 (2), 1-28. Recuperado de h t t p : / / w w w. red a ly c.org / pdf/447/44740211.pdf

Quintero, V. (1995). Evaluación de proyectos sociales: construcción de indicadores. Cali: FES.

República de Colombia. Departamento Nacional de Planeación. (2014). Plan Nacional de Desarrollo 2014-2018. Recuperado de https://https://colaboracion.dnp.gov.co/CDT/PND/PND\%20 2014-2018\%20Tomo\%201\%20internet. pdf

República de Colombia. Congreso de Colombia (1992). Por la cual se organiza el servicio público de la Educación Superior. [Ley 30]. DO: 40.700. Recuperado de http://www.cna.gov.co/1741/articles-186370_ley_3092.pdf

República de Colombia. Ministerio de Educación Nacional. (2013). Estructura de indicadores de perfilación y caracterización, para las Instituciones de Educación Superior en Colombia. Bogotá, D.C.: Mineducación.

Salamanca, F. (1995). Formulación y Evaluación de Proyectos Sociales. Curso de post grado del Programa Global de Formación en Población y Desarrollo. CELADE.

Salinas, F. y Osorio, L. (2012). Emprendimiento y Economía Social, oportunidades y efectos en una sociedad en transformación. Revista de Economía Pública, Social y Cooperativa, 75, 128151. Recuperado de http://www.redalyc. org/articulo.oa?id=17425798008
Shahidur, K., Gayatri, K. y Hussain, S. (2010). Impact Evaluation: Cuantitative methods and practices. Washington: The World Bank. Recuperado de http:// documents.worldbank.org/curated/ en/650951468335456749/pdf/520990P UB0EPI1101Official0Use0Only1.pdf

Valadez, J. y Bamberger, M. (1994). Monitoring and Evaluating Social Programs in Developing Countries. Washington, D.C: EDI Development Studies.

Valdés, M. (2008). La evaluación de impacto de proyectos sociales: Definiciones y conceptos. Recuperado de https://www. mapunet.org/documentos/mapuches/ Evaluacion_impacto_de_proyectos_sociales.pdf

Wooldridge, J. (2008). Introducción a la econometría: un enfoque moderno. Madrid: Thomson Paraninfo.

Carolina Rincón Zapata es Economista, especialista en Finanzas con énfasis en Banca de Inversión. Magister en Economía y Docente tiempo completo de la Facultad de Ciencias Económicas y Administrativas en la Universidad Católica de Oriente (Colombia)

Adriana Pérez Cadavid es Ingeniera Industrial, especialista en Gerencia, Especialista en Pedagogía y Didáctica con Magister en Educación y Docente tiempo completo de la Facultad de Ciencias Económicas y Administrativas en la Universidad Católica de Oriente (Colombia) 